\title{
An Ethical Argument for Professional Regulation and Regionalization of Care in Pediatric Cardiology and Cardiac Surgery
}

\author{
Angira Patel ${ }^{1}$ (D) $\cdot$ Rupali Gandhi ${ }^{2}$
}

Received: 27 January 2020 / Accepted: 18 February 2020 / Published online: 20 March 2020

๑) Springer Science+Business Media, LLC, part of Springer Nature 2020

Survival for children with congenital heart disease (CHD) has improved significantly over the last three decades. From 1979 to 1993 , only $67 \%$ percent of infants with the most severe forms survived to one year of age, whereas from 1994 to 2005, 82.5\% survived [1]. Nevertheless, data show that mortality improvements for infants with CHD are not uniform across institutions and centers that perform fewer surgeries typically have higher mortality rates [2].

Recently, a high-profile article in the New York Times highlighted another pediatric cardiac surgery center in the USA forced to suspend surgeries due to high mortality in infants undergoing surgical repair for CHD [3]. In the USA, there is no mandated public reporting or a central authority that regulates the number of cardiac congenital procedures or surgeries a hospital must perform to maintain a program. Some European countries have implemented regulations that limit the total number of programs that can perform CHD surgeries to ensure good clinical outcomes and quality of care [4].

Although much has been written on this topic, the suspension or closure of pediatric cardiac surgery programs for poor performance is a problem that should be contextualized in the sphere of medical ethics using principles of non-maleficence, respect for autonomy, and justice. One of the first tenets of medicine is "non-maleficence" or "do no harm." This can only be practiced when physicians use informed consent and shared decision-making with patients.

Informed consent demonstrates respect for autonomy and is the process that physicians and patients practice when determining a course of treatment. The elements that must

Angira Patel

anpatel@1uriechildrens.org

1 Ann and Robert H Lurie Children's Hospital of Chicago, Northwestern University Feinberg School of Medicine, 225 E. Chicago Ave., Box 21, Chicago, IL 60611-2991, USA

2 Advocate Children's Hospital, 4440 West 95th Street, Oak Lawn, IL 60453-2699, USA be present for informed consent to be valid are: (1) disclosure of medical information (burdens, risks, benefits, alternatives) by the physician, (2) competency of the patient or surrogate decision-maker to understand, process, and make decisions, and (3) lack of coercion. In pediatric cardiac surgery programs, before informed consent can be practiced, physicians need a system by which they can stay abreast of their current institutional outcomes and how they compare nationwide. It is reasonable to consider whether informed consent was fully practiced by these programs. Despite outcomes that were beyond what is acceptable morbidity and mortality, some programs continued to operate on children. In addition, parents were not necessarily informed that the surgical outcomes had been declining and not privy to important information that directly impacts the care of their child [5, 6]. If parents lack full knowledge of the risks that they were putting upon their children, the consent that parents give for surgery could not have been truly informed. Whenever a physician engages in an informed consent discussion, the physician is supposed to discuss the risks, benefits, and alternatives that "the reasonable patient" would want to know-i.e., what would a reasonable person want to know about this procedure and alternatives [7]? Although physicians may disagree over disclosing differences in outcomes that are within acceptable variation or risks that are in a gray area (either not very serious or not very common), the increased morbidity and mortality at these surgical programs was not trivial nor a one-off. One can assume that a reasonable parent would have wanted to know about them.

Furthermore, when medical care and decision-making are complex, the process of shared decision-making must occur whereby there is an in-depth conversation between physicians and patients. In this conversation, the patient has an opportunity to actively participate in choosing the best treatment using available evidence and based on their own specific preferences. In pediatrics, parents act as proxy decision-makers for their child using the best-interest standard. With infants undergoing surgery for CHD, conversations 
should include information sharing such as details of the surgical and medical interventions, institutional outcomes, current standard of care, expected morbidity and mortality, and available resources at the institution incase complications occur. This shared decision-making process involves all the components of informed consent but is not a one-time event and must be revisited as the clinical situations evolve.

Finally, the ethical principle of justice argues for medical professionals to address disparities in care, access to care, and inherent structural inequalities. When surgical institutions can continue to exist while providing substandard care, the disparities in care can impact the most vulnerable and families with fewer resources. The resourceful and highly educated parents may be able to seek out information independently and may be able to transfer care elsewhere. These parents may have access to second opinions, friends and family members who are in the medical field, or an inside source who can help them navigate the system. Those who continue to get their treatment at substandard places are often unaware that the care is subpar, or they do not have the means to make any other choice. Allowing such a dual tiered system to exist is ethically troublesome, antitheses to the goals of medicine, and further increases structural inequities.

Admittedly, when infants undergo complex surgery for critical CHD, not all surgeries have perfect outcomes. There will be residual disease, complications, and deaths despite having a program that delivers excellent clinical care. It is also naive to believe that a poor outcome is the direct result of poor surgical skills; pre-operative and post-operative management, expertise in interventional cardiology, electrophysiology, imaging, and cardiac anesthesia are also needed to provide the best care possible. Some patients with genetic or other co-morbidities are higher risk and expected to have different outcomes than a standard-risk patient. Surgical innovation involves inherent risks and should not be completely stifled either [8]. Finally, how reliably outcomes and quality are measured and how metrics are reported remain critically important. While acknowledging that there is a complex interaction of factors that leads to a poor surgical outcome, we as physicians must always remember that patients come first. The difference between expected (normal-range) mortality and the 4-5 times increased mortality that has been reported in the media falls outside the "gray zone."

The pediatric cardiology community recognizes the need for professional regulation and has advocated for multiple solutions including improving the process of shared decision-making through guided questions, transparency through creating databases that report outcomes, and regionalization of care through dedicated centers of excellence [9-12]. Pediatric cardiologists and surgeons must also be given an avenue to voice their concerns about problematic outcomes at their own institutions. Cultural and administrative barriers to open discussion about poor outcomes must be lifted to avoid physician moral distress and provide patients with honest answers.

To enact these solutions, key stakeholders will need to collaborate including physicians, hospitals, professional organizations, insurance companies, community members, families, and legislators. Professional organizations are in the early stages of tackling this problem by promoting transparency with regards to clinical and case volumes, disease complexities, and accurately defining outcomes. Insurance companies are often the drivers that determine where specialized care will occur, and financial motivators to promote care at larger volume centers could be beneficial to the patients, as well as insurers' bottom lines. Community members and families must also be involved as they provide an important perspective on balancing risk and benefits. Finally, legislators who can help enact policy will need to be involved to provide infrastructure needed to promote regionalized care.

When there is direct patient harm, we must look at the reasons, create concrete solutions, and regulate our own profession. As a medical community but also a moral community, we must call on our oath to be responsible to and for our young patients so that all children with congenital heart disease receive the best care possible.

\section{Compliance with Ethical Standards}

Conflict of interest All the authors have no conflicts of interest to disclose.

\section{References}

1. Oster ME, Lee KA, Honein MA et al (2013) Temporal trends in survival among infants with critical congenital heart defects. Pediatrics 131(5):1502-1508

2. Pasquali SK, Li JS, Burstein DS et al (2012) Association of center volume with mortality and complications in pediatric heart surgery. Pediatrics 129(2):e370-e376

3. Doctors were Alarmed: 'Would I Have My Children Have Surgery Here?' (2019) https://www.nytimes.com/interactive/2019/05/30/ us/children-heart-surgery-cardiac.html

4. Daenen W, Lacour-Gayet F, Aberg T et al (2003) Optimal structure of a congenital surgery department in Europe: by EACTS congenital heart disease committee. Eur J Cardio-Thorac Surg 24(3):343-351

5. Troubled Children's Hospital is Sued Over Toddler Who Died After Surgery (2019) https://www.nytimes.com/2019/12/05/us/ heart-surgery-death-lawsuit.html

6. Heartbroken (2018) https://projects.tampabay.com/projects/2018/ investigations/heartbroken/all-childrens-heart-institute/

7. Derse AR (2017) Flying too close to the sun: lessons learned from the judicial expansion of the objective patient standard for informed consent in Wisconsin. J Law Med Ethics 45:51-59 
8. Collins RT, Shin AY, Hanley FL (2020) Sacrificing the future for the sake of the present. Ann Surg 271(2):225-226

9. Pasquali SK, Dimick JB, Ohye RG (2015) Time for more unified approach to pediatric health care policy? The case of congenital heart care. JAMA 324(10):1689-1690

10. Backer CL, Karamlou T, Welke KF (2019) More evidence for regionalization. JACC 74(23):2919-2920

11. Welke KF, Pasquali SK, Lin P et al (2019) Regionalization of congenital heart surgery in the United States. Semin Thorac Cardiovasc Surg. https://doi.org/10.1053/j.semtcvs.2019.09.005
12. Backer CL, Pasquali SK, Dearanhi JA (2020) Improving national outcomes in congenital heart surgery: the time has come for regionalization of care. Circulation. https://doi.org/10.1161/ CIRCULATIONNAHA.119.045542

Publisher's Note Springer Nature remains neutral with regard to jurisdictional claims in published maps and institutional affiliations. 